\title{
Contribuição ao estudo farmacognóstico de Mikania laevigata Sch. Bip. ex Baker (guaco), visando o controle de qualidade da matéria-prima
}

\author{
Jane M. Budel, ${ }^{*}, 1,2$ Márcia R. Duarte, ${ }^{1}$ Indianara Kosciuv, ${ }^{3}$ \\ Tatiane B. de Morais, ${ }^{2}$ Lilian P. Ferrari ${ }^{2}$ \\ ${ }^{1}$ Laboratório de Farmacognosia, Universidade Federal do Paraná, Av. Pref. Lothário Meissner, \\ 632, Jardim Botânico, 80210-170 Curitiba-PR, Brasil \\ ${ }_{2}^{2}$ Laboratório de Farmacognosia, Unibrasil, Rua Konrad Adenauer, 442, Tarumã, 82820-540 \\ Curitiba-PR, Brasil \\ ${ }^{3}$ Laboratório de Farmacobotânica, Uniguaçu, Rua Padre Saporiti, 717, Rio D’Areia, 84600-000 \\ União da Vitória-PR, Brasil
}

\begin{abstract}
RESUMO: O gênero Mikania, pertencente à família Asteraceae e à tribo Eupatoriae, tem cerca de 430 espécies distribuída principalmente na América do Sul. No Brasil, o gênero está representado por aproximadamente 171 espécies. Várias espécies do gênero Mikania de hábito trepador recebem a denominação vulgar de "guaco". Mikania laevigata Sch. Bip. ex Baker, conhecida como "guaco", "guaco-de-casa" e "guaco-do-mato", é uma espécie nativa do sul do Brasil. Suas folhas são empregadas na medicina tradicional como expectorante e como anti-reumáticas. É muito semelhante morfologicamente com M. glomerata, que é a espécie oficializada pela Farmacopéia Brasileira I. Alguns estudos de M. laevigata comprovaram atividades farmacológicas como, antiinflamatória, antimutagênica, antimicrobiana e antiulcerogênica. Objetivou-se estudar a morfologia externa e a anatomia do caule e da folha de M. laevigata, com a finalidade de fornecer dados farmacognósticos referentes à identificação e à diferenciação dessa espécie das demais Mikania, visando o controle de qualidade da matéria-prima. $\mathrm{O}$ material botânico foi submetido às microtécnicas fotônicas e eletrônicas de varredura usuais. As características morfoanatômicas descritas para a folha e o caule de M. laevigata auxiliam na identificação da espécie.
\end{abstract}

Unitermos: Asteraceae, Mikania laevigata, controle de qualidade.

\begin{abstract}
Contribution to the pharmacognostic study of Mikania laevigata Sch. Bip. ex Baker "Guaco" for the quality control". The genus Mikania, belonging to the family Asteraceae and tribe Eupatoriae, has about 430 species mainly distributed in South America. In Brazil, the genus is represented by approximately 171 species. Various members of Mikania are lianas and commonly called "guaco". Mikania laevigata Sch. Bip. ex Baker, known as "guaco", "guaco-decasa" and "guaco-do-mato", is native to the South of Brazil. Its leaves are used in folk medicine as expectorant and antirheumatic. It is morphologically similar to M. glomerata, whose monograph is included in the Brazilian Pharmacopoeia 1st Ed. Some studies on M. laevigata have demonstrated pharmacological activities, such as anti-inflammatory, antimutagenic, antimicrobial and antiulcerogenic. This work has aimed to study the morpho-anatomy of the stem and the leaf of $M$. laevigata, in order to supply pharmacognostic information related to the identification of this species and distinction from other Mikania for quality control purposes. The botanical material was prepared according to standard light and scanning electronic micro techniques. The morphoanatomical characters described for the stem and the leaf contribute to the identification of this species.
\end{abstract}

Keywords: Asteraceae, Mikania laevigata, quality control.

\section{INTRODUÇÃO}

O gênero Mikania, pertencente à família Asteraceae e à tribo Eupatoriae, tem cerca de 430 espécies distribuída principalmente na América do Sul. No Brasil, o gênero, com 171 espécies, ocorre de norte a sul, tendo sua principal área de dispersão nos estados de Minas Gerais, Rio de Janeiro e São Paulo. (Oliveira, 1972; Ritter \& Miotto, 2005).

Muitos trabalhos se detêm na determinação 
de compostos químicos, principalmente flavonóides (Aguinaldo et al., 2003), terpenos (Fabri et al., 1997; Lobitz et al., 1997, 1998; Veneziani et al, 1999; Reis et al., 2003; Nunez et al., 2004; Ohkoshi et al., 2004; Reis et al., 2008), lactonas sesquiterpênicas (Cuenca et al, 1993; Zamorano et al., 1994; Bardon et al., 1996; Veneziani et al., 1999; Rüngeler et al., 2001) e cumarinas (Biavati et al., 2004; Santos et al., 2006; Taleb-Contini et al., 2006; Saúde-Guimarães \& Faria, 2007; Corrêa et al., 2008; Silva et al., 2008; Bolina et al., 2009).

A ação farmacológica de extratos ou de substâncias isoladas de Mikania têm sido investigada para diversas espécies, como as atividades antiinflamatória de $M$. involucrata Hook. et Arn. e M. hirsutissima DC. (Suyenaga et al, 2002), bactericida, antifúngica e hipoglicêmica de $M$. micranta Kunth (Lentz et al., 1998; Barbosa-Filho et al., 2005), bactericida, anti-ofídica, antialérgica, antiinflamatória, anti-diarréica, inibidora da MAO, antimalárica e broncodilatadora de $M$. glomerata Spreng. (Fierro et al., 1999; Holetz et al., 2002; Soares et al., 2002; Do Amaral et al., 2003; Falcão et al., 2005; Maiorano et al., 2005; Salgado et al., 2005; Santos et al., 2006; Botsaris, 2007), anti-carcinogênica, analgésica, antiulcerogênica e anticonvulsivante de $M$. cordata (Burm. f.) B.L. Rob. (Bishayee \& Chaterjee, 1994; Paul et al., 2000; Ahmed et al, 2001; Quintans-Júnior et al., 2008), antiprotozoária de $M$. cordifolia (L. f.) Willd. (Arias et al., 1995; Muelas-Serrano et al., 2000), antibacteriana de $M$. lanuginosa DC. (Silva et al., 2002) e anti-Tripanosoma cruzy de M. obtusata (Saúde-Guimarães \& Faria, 2007).

Diversas espécies do gênero Mikania de hábito trepador recebem a denominação vulgar de guaco, a exemplo de $M$. cordifolia, M. laevigata Sch. Bip. ex Baker, M. glomerata, M. scandens Willd., M. officcinalis Mart., M. opifera DC. (Barroso, 1958; Angely, 1965; Corrêa, 1984; Oliveira et al., 1999; Oliveira et al., 2000; Ritter \& Miotto, 2005).

Mikania laevigata, conhecida como guaco, guacode-casa e guaco-do-mato, é uma espécie nativa do sul do Brasil. Suas folhas são empregadas na medicina tradicional como expectorante e anti-reumáticas (Alice et al., 1995). É muito semelhante morfologicamente com M. glomerata Spreng., que é a espécie oficializada pela Farmacopéia Brasileira I (Silva, 1929). Alguns estudos de $M$. laevigata comprovaram atividades farmacológicas como, antiinflamatória (Suyenaga et al., 2002), antimutagênica (Fernandes \& Vargas, 2003), antimicrobiana e alelopática (Duarte et al., 2004; Baratto et al., 2008) e antiulcerogênica (Bighetti et al., 2005).

Objetivou-se estudar a morfologia externa e a anatomia do caule e da folha de $M$. laevigata, com a finalidade de fornecer dados farmacognósticos referentes à identificação e à diferenciação dessa espécie das demais Mikania.

\section{MATERIAL E MÉTODOS}

O material vegetal foi coletado em dezembro de 2003 na Fazenda São Maximiano, situada na região da Serra do Sudoeste, em Guaíba, Rio Grande do Sul $\left(30^{\circ}\right.$ $10^{\prime} \mathrm{S}$ e $\left.51^{\circ} 20^{\prime} \mathrm{W}, 27 \mathrm{~m}\right)$. Foi realizada a confecção da exsicata, que foi identificada por taxonomista e se encontra depositada no Instituto de Ciências Naturais (ICN) da Universidade Federal do Rio Grande do Sul sob o número ICN 51062 .

A partir de $5 \mathrm{~cm}$ do ápice da planta, o caule e as folhas de $M$. laevigata foram fixados em solução de FAA 70 (Johansen, 1940) e armazenados em álcool etílico a 70\% (Berlyn \& Miksche, 1976). Lâminas semipermanentes foram preparadas com o material seccionado nos sentidos transversal e longitudinal, à mão livre, e submetido à coloração de azul de astra e fucsina básica (Roeser, 1972) ou de azul de toluidina (O'Brien et al., 1964).

Utilizando-se do material previamente fixado e armazenado em álcool etílico a $70 \%$, as lâminas permanentes foram preparadas empregando-se a técnica de inclusão em glicol metacrilato (Feder \& O'Brien, 1968). O caule e a folha de $M$. laevigata foram seccionados no plano transversal em micrótomo, obtendo-se cortes de 7 a $9 \mu \mathrm{m}$. Os cortes foram hidratados, distendidos em lâminas e secos em mesa térmica a $40{ }^{\circ} \mathrm{C}$. Para coloração empregou-se azul de astra e fucsina básica (Brito \& Alquini, 1996).

Para a análise microquímica foram utilizados os seguintes reativos: solução de floroglucina clorídrica para verificação de lignina (Foster, 1949), Sudan III para compostos lipofílicos (Sass, 1951), cloreto férrico para compostos fenólicos (Johansen, 1940), lugol para amido (Berlyn \& Miksche, 1976) e ácido sulfúrico para verificação da natureza química dos cristais (Oliveira \& Akisue, 1991).

A microscopia eletrônica de varredura da superfície foliar e caulinar foi realizada em alto vácuo e para tal procedimento, as amostras foram desidratadas em série etanólica crescente e pelo ponto crítico de $\mathrm{CO}_{2}$ e, após montagem em suporte, submetidas à metalização com ouro (Souza, 1998).

\section{RESULTADOS}

Mikania laevigata (Figura 1) é um sub-arbusto trepador que apresenta caule cilíndrico e lenhoso. A região dos nós é, freqüentemente, acompanhada por folhas de disposição oposta, estas se caracterizam por serem ovadas a oblongo-lanceoladas, de ápice acuminado e base obtusa, arredondada ou subcordiforme, de margem inteira, pecioladas, e por apresentarem base trinervada.

Em relação aos caracteres anatômicos, a lâmina foliar apresenta, em vista frontal, células de contorno de ondeado a sinuoso, de paredes anticlinais levemente espessadas (Figuras 2 e 3) A folha apresenta estômatos do tipo anomocíticos e anisocíticos somente na face abaxial, 
caracterizando a folha como hipoestomática (Figura 2) e estes se localizam no mesmo nível das demais células epidérmicas. Sobre o sistema de revestimento, ocorre presença de cutícula delgada e levemente estriada (Figura $4)$.

Evidencia-se tricomas glandulares pluricelulares unisseriados, curvos, formados por cerca de seis células (Figura 5), além de tricoma glandular pluricelular bisseriado (Figuras 6 e 7). Esses anexos epidérmicos aparecem em depressão na epiderme (Figuras 5, 6 e 7).

O mesofilo é do tipo dorsiventral, sendo constituído por cerca de três camadas de parênquima paliçádico e aproximadamente oito estratos de parênquima esponjoso, que evidencia pequenos espaços intercelulares. Subjacente à epiderme adaxial, observa-se uma camada contínua de células de formato retangular, alongadas no sentido periclinal e maiores que as células epidérmicas (Figura 8).

Feixes vasculares colaterais estão mergulhados na região mediana do mesofilo e aparecem envoltos por uma bainha parenquimática e freqüentemente estão associados a dutos secretores, estes são formados por cerca de sete células em estrato único (Figura 9).

A nervura central, em secção transversal, apresenta-se biconvexa. A epiderme é uniestratificada, recoberta por cutícula delgada e levemente estriada. O colênquima é do tipo angular e é representado por cerca de quatro estratos em ambas as faces. Mergulhado no parênquima fundamental, cerca de cinco feixes vasculares do tipo colateral, dispostos em arco aberto, são observados. Calotas de fibras esclerenquimáticas, aposta ao floema,
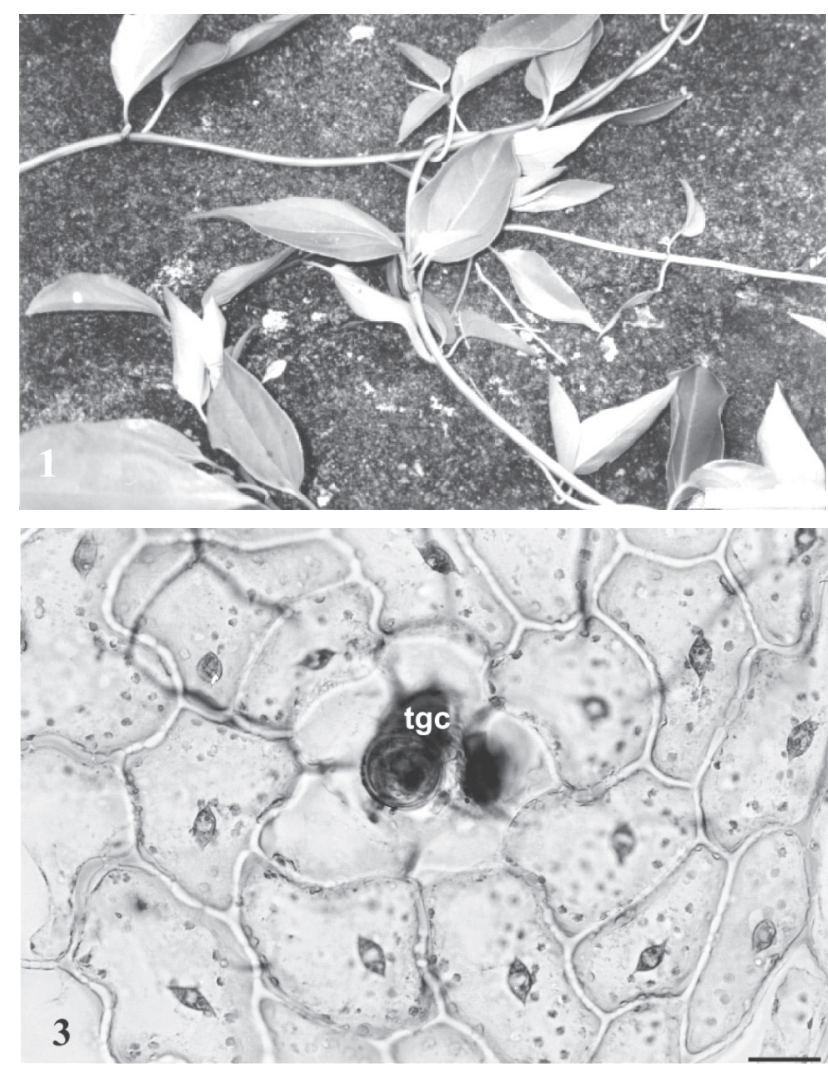

podem ser encontradas. Dutos secretores são observados nas proximidades do feixe vascular e apresentam características semelhantes às anteriormente descritas. $\mathrm{O}$ xilema é constituído por elementos traqueais dispostos em fileira (Figura 10).

O pecíolo, em secção transversal (Figura 11), tem formato praticamente plano-convexo, e evidencia epiderme uniestratificada. Subjacentemente à epiderme, observam-se cerca de seis camadas de colênquima angular. Cerca de sete feixes vasculares, do tipo colateral, em arco aberto, estão mergulhados no parênquima fundamental.

O caule, em secção transversal, apresenta formato circular. A epiderme é uniestratificada e as células são alongadas no sentido periclinal e revestidas por cutícula moderadamente delgada. Adjacentemente à epiderme, cerca de seis camadas de colênquima angular são observados. Limitando internamente $\mathrm{o}$ córtex, observa-se um estrato de células parenquimáticas contendo amiloplastos, constituindo a bainha amilífera. Nas proximidades desta, em direção aos feixes vasculares, dutos secretores de estrato único, formados por cerca de oito células podem ser encontrados. O cilindro vascular é constituído por floema em direção centrífuga e xilema formado centripetamente, estabelecendo uma região medular. Calotas de fibras perivasculares são encontradas na região próxima ao sistema floemático. O parênquima medular compõe-se de células de diversos tamanhos, de parede delgada e evidencia vários dutos secretores (Figura 12).

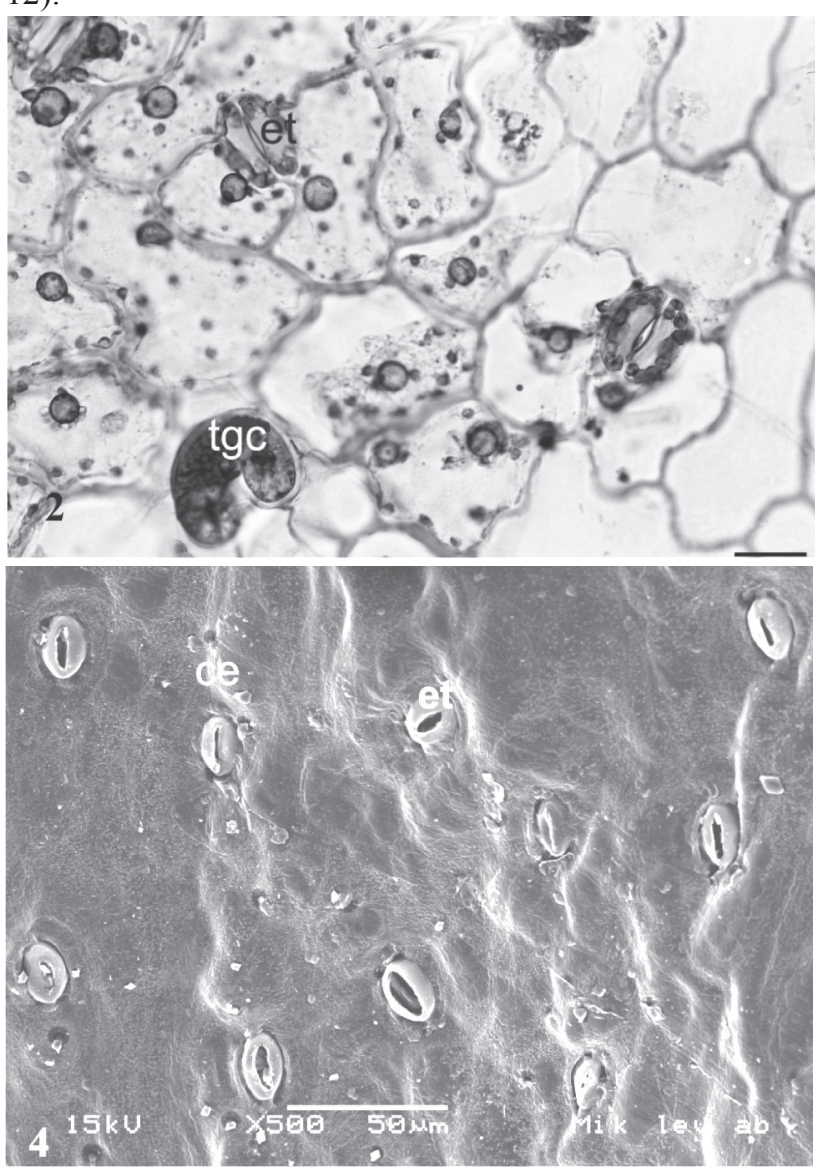



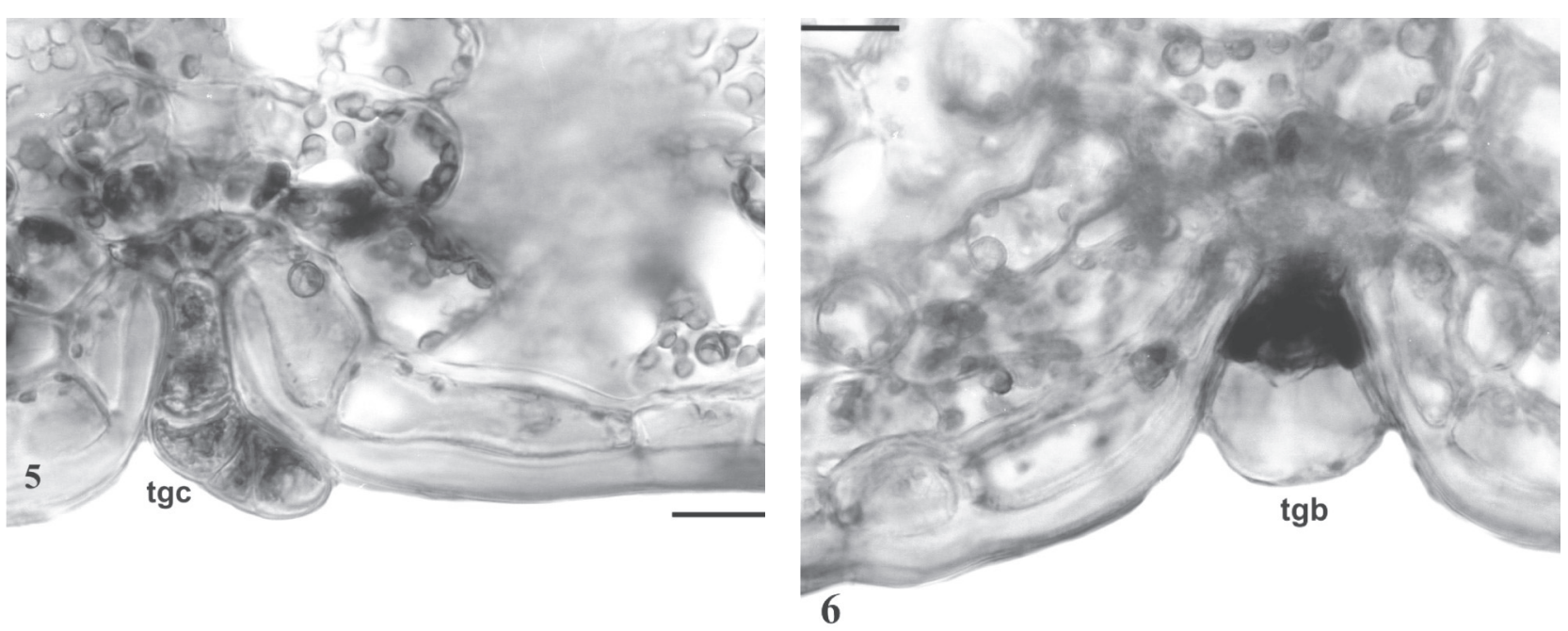

Figuras 1-6. Mikania laevigata Sch. Bip. ex Baker. 1. Aspecto geral; 2. Vista frontal da epiderme foliar, face abaxial, mostrando o contorno das células epidérmicas, estômato anisocítico (et) e tricoma glandular pluricelular curvo (tgc); 3 . Vista frontal da epiderme foliar, face adaxial, mostrando o contorno das células e tricoma glandular pluricelular curvo; 4. Vista frontal da epiderme foliar, indicando estômatos e cutícula levemente estriada (ce); 5. Secção transversal da folha, evidenciando tricoma glandular pluricelular curvo; 6. secção transversal da folha, mostrando tricoma glandular pluricelular bisseriado (tgb). Barra $=20 \mu \mathrm{m}$.
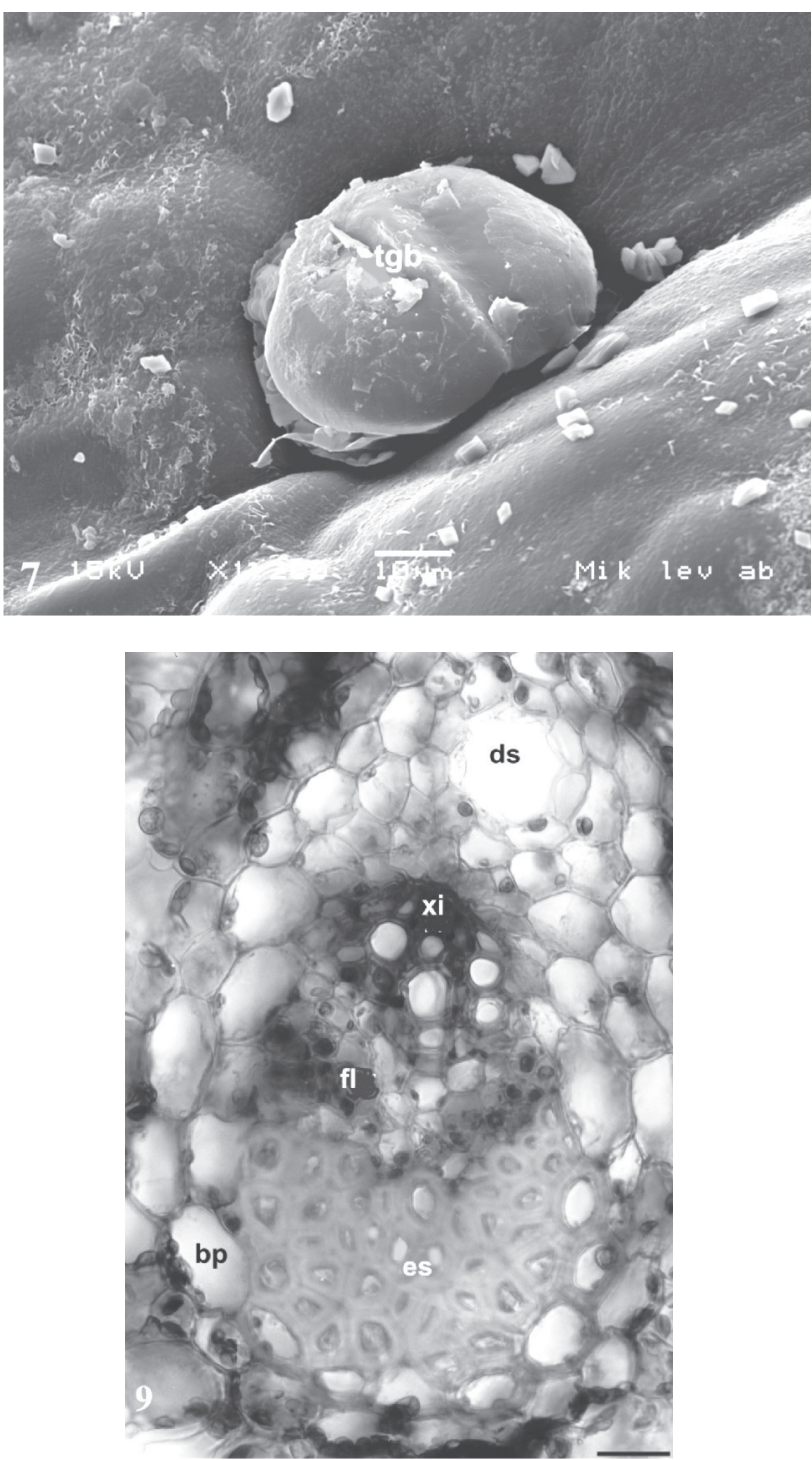
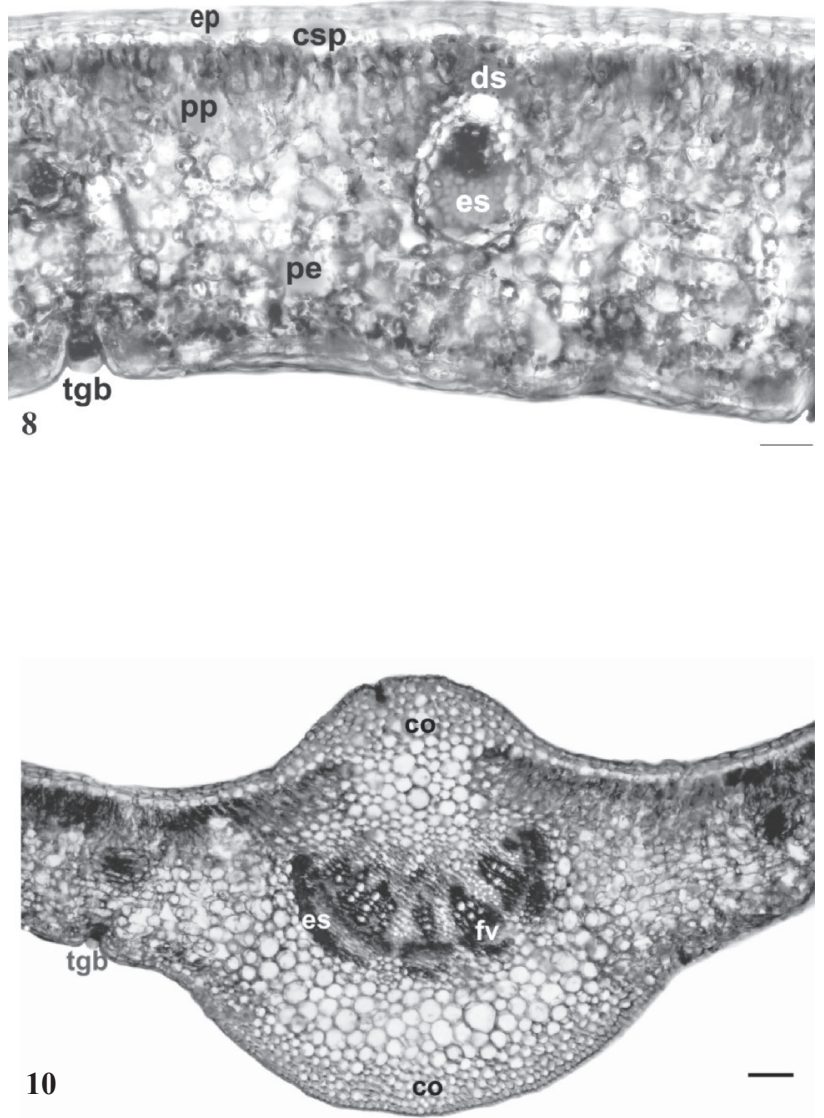

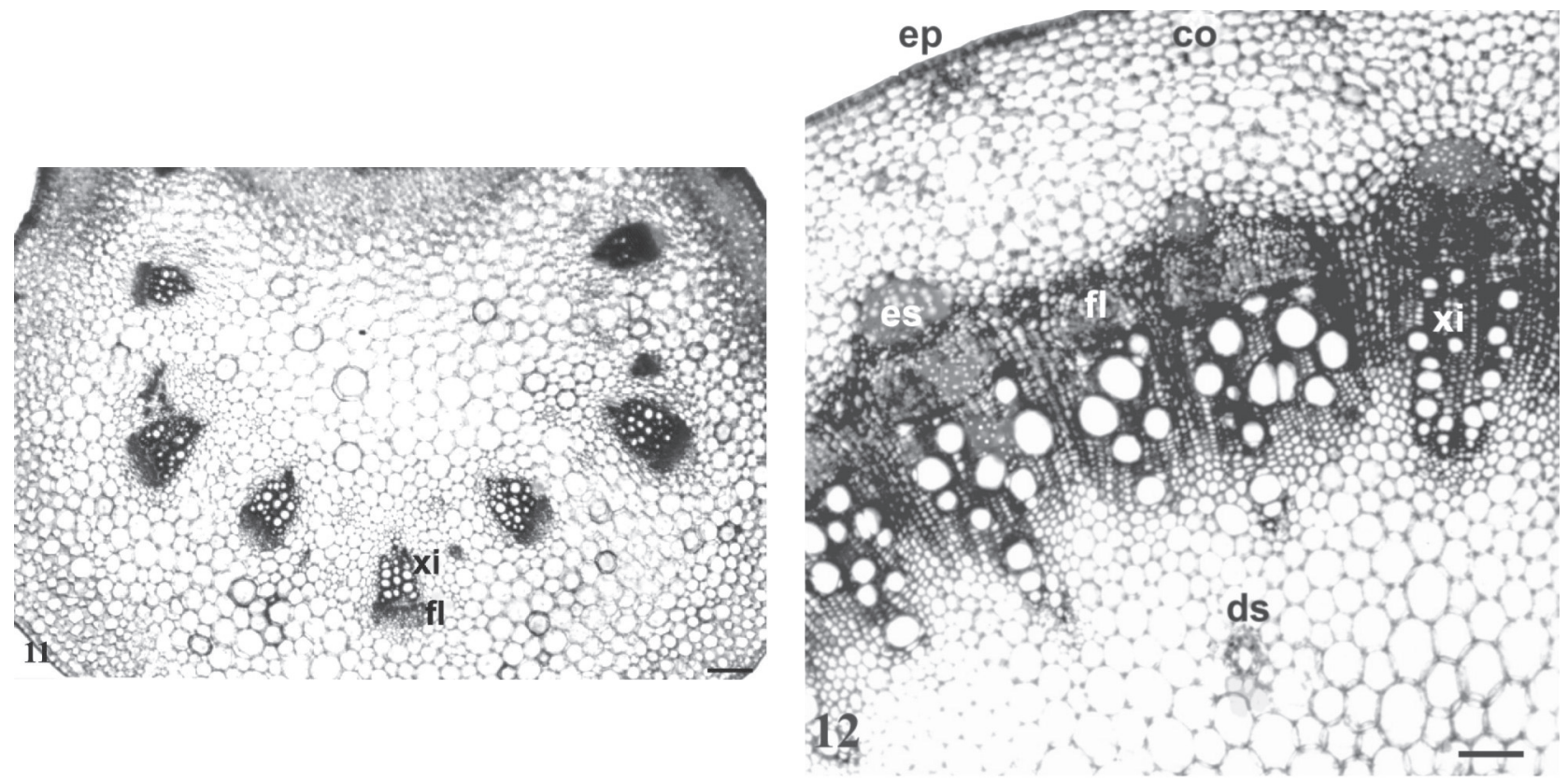

Figuras 7-12. Mikania laevigata Sch. Bip. ex Baker.7. Vista frontal da epiderme foliar, indicando tricoma glandular pluricelular bisseriado (tgb); 8.Secção transversal da folha, onde pode ser observado mesofilo dorsiventral, calota de fibras perivasculares (es), camada subepidérmica (csp), epiderme (ep), duto secretor (ds), parênquima esponjoso (pe), parênquima paliçádico (pp), tricoma glandular pluricelular bisseriado; 9. Detalhe da figura anterior, mostrando bainha parenquimática (bp), calota de fibras perivasculares, duto secretor, floema (fl) e xilema (xi);10. Secção transversal da folha, mostrando formato biconvexo da nervura central, calota de fibras perivasculares, colênquima angular (co), epiderme, calota de fibras perivasculares e feixe vascular colateral (fv); 11. Secção transversal do pecíolo, indicando organização dos feixes vasculares, floema e xilema; 12. Secção transversal do caule, onde se notam epiderme, colênquima angular, calota de fibras perivasculares, floema, xilema e duto secretor. Barra $=20 \mu \mathrm{m}(9) ; 50 \mu \mathrm{m}(8,10,11)$; $100 \mu \mathrm{m}(12)$.

\section{DISCUSSÃO}

Os caracteres morfológicos externos estão de acordo com as informações referentes à espécie (Oliveira et al., 1986a, b; Alice et al., 1995). Quanto ao formato da folha, M. confertissima Sch. Bip. ex Baker é muito parecida com M. laevigata, podendo causar confusão (Oliveira et al., 1986a) .O contorno das células epidérmicas, em vista frontal, encontrados na folha de $M$. laevigata concorda com o constatado para essa espécie (Oliveira et al., 1986a) e para M. glomerata (Neves \& Sá, 1991).

Em secção transversal, $M$. laevigata mostrou epiderme e camada subepidérmica, esta com células retangulares e um pouco maiores que a epiderme e presença de cutícula delgada e levemente estriada. Esses caracteres vão ao encontro dos dados relatados para a espécie em estudo (Oliveira et al., 1986a) e para M. glomerata (Neves \& Sá, 1991).

A descrição de tricomas constitui característica importante na diagnose do fármaco, principalmente quando o fármaco apresenta-se fragmentado ou pulverizado (Oliveira et al., 1993). No estudo em questão, M. laevigata evidenciou dois tipos de tricomas glandulares, a saber, pluricelulares unisseriados, curvos, formados por cerca de seis células, e pluricelulares bisseriados, localizados em depressão na epiderme. Os primeiros, foram citados no trabalho de Oliveira et al. (1986a), não havendo menção do segundo tipo de tricoma para $M$. laevigata.

Tricomas glandulares pluricelulares bisseriados, similares aos encontrados neste estudo foram encontrados em M. glomerata e classificados como capitados por Neves \& Sá (1991) e como peltados por Milan et al. (2006). Tricomas curvos são característicos do gênero Mikania (Oliveira et al., 2000) e foram citados para $M$. conferta (Oliveira et al., 1999), M. glomerata (Neves \& Sá, 1991), M. malacolepsis (Rodrigues et al., 1996) e M. cordifolia (Oliveira et al., 2000). Esses tricomas foram mencionados como simples pluricelulares do tipo claviforme para $M$. glomerata (Neves \& Sá, 1991). Tricomas tectores não encontrados no estudo em questão, foram mencionados em M. cordifolia e M. malacolepsis e descritos como unisseriados pluricelulares terminado em ponta (Rodrigues et al., 1996; Oliveira et al., 2000) e podendo assumir a forma de ampola em M. conferta (Oliveira et al., 1999).

Segundo Metcalfe \& Chalk (1950), os estômatos podem ser anomocíticos e anisocíticos na família Asteraceae, com predominância do primeiro tipo, e podem ocorrer em ambas as faces epidérmicas. Estas mesmas categorias foram descritas para diversas espécies do gênero Mikania (Alice et al., 1995; Rodrigues et al., 
1996; Neves \& Sá, 1991; Oliveira et al., 1999; Oliveira et al., 2000; Oliveira et al., 2005; Milan et al., 2006).

A folha de $M$. laevigata evidenciou a presença de estômatos anomocíticos e anisocíticos somente na face abaxial, caracterizando a folha como hipoestomática. Esta mesma característica foi observada em M. glomerata (Neves \& Sá, 1991; Oliveira et al., 2005) e em M. hirsutissima (Oliveira et al., 2005).

A organização do parênquima fotossintetizante é do tipo dorsiventral em $M$. laevigata, característica que vai ao encontro dos relatos de diversas espécies do gênero (Neves \& Sá, 1991; Alice et al., 1995; Rodrigues et al., 1996; Oliveira et al., 1999; Oliveira et al., 2000; Oliveira et al., 2005). Milan et al. (2006), relatam que a organização do mesofilo de $M$. glomerata é dorsiventral, mas pode variar de acordo com as diferentes regiões da folha. Adicionalmente, Neves \& Sá (1991), descrevem o parênquima paliçádico de $M$. glomerata constituído por células braciformes em forma de $\mathrm{H}, \mathrm{V}$ e Y.

$\mathrm{Na}$ nervura central da espécie em estudo, o formato biconvexo, a disposição dos feixes vasculares colaterais, a presença de colênquima angular, de calota de fibras perivasculares e de dutos secretores são características que coincidem com as relatadas para $M$. laevigata (Oliveira et al., 1986a), M. glomerata (Neves \& Sá, 1991; Oliveira et al., 2005; Milan et al., 2006), M. malacolepsis (Rodrigues et al., 1996), M. conferta (Oliveira et al., 1999) e M. cordifolia (Oliveira et al., 2000).

$\mathrm{O}$ caule de $M$. laevigata mostra contorno circular, característica observada para esta espécie (Oliveira et al., 1986b) e também para M. glomerata (Neves \& Sá, 1991), entretanto, M. malacolepsis (Rodrigues et al., 1996) e M. cordifolia (Oliveira et al., 2000) evidenciaram contorno hexagonal para o caule.

Segundo Oliveira et al. (2000), caules desenvolvidos de Mikania podem evidenciar na região cortical a presença de anel esclerenquimático, característica freqüente em Mikanias trepadoras. Todavia esse anel não foi observado em M. laevigata.

Dutos secretores próximos aos feixes vasculares e também na medula do caule de $M$. laevigata foram encontrados, essas informações concordam com o descrito para várias espécies do gênero (Oliveira et al., 1986b; Neves \& Sá, 1991; Oliveira et al., 1999), contudo, dutos não foram encontrados na região medular de M. cordifolia (Oliveira et al., 2000).

Usualmente a morfologia do cristal, bem como a sua distribuição no órgão é conservada dentro de um específico táxon (Franceschi \& Horner Junior, 1980). Nesse contexto, nos órgãos analisados de M. laevigata não foram encontrados cristais. A ausência destes parece ser característica do gênero (Neves \& Sá, 1991; Rodrigues et al., 1996; Oliveira et al., 1999; Oliveira et al., 2000; Oliveira et al., 2005).

\section{AGRADECIMENTOS}

Os autores agradecem ao taxonomista Prof. Dr. N. I. Matzenbacher pela identificação da espécie e ao Centro de Microscopia Eletrônica da Universidade Federal do Paraná pelas eletromicrografias.

\section{REFERÊNCIAS}

Aguinaldo AM, Padolina WG, Abe F, Yamauchi T 2003. Flavonoids from Mikania cordata. Biochem Syst Ecol 31: 665-668.

Ahmed M, Rahaman MT, Alimuzzaman M, Shilpi JA 2001. Analgesic sesquiterpene dilactone from Mikania cordata. Fitoterapia 72: 919-921.

Alice CB, Siqueira NCS, Mentz LA, Silva GAAB, José KFD 1995. Plantas medicinais de uso popular: atlas farmacognóstico. Canoas: Ulbra.

Angely J 1965. Flora analitica do Paraná. São Paulo: Phyton. Arias AR, Ferro E, Inchausti A, Ascurra M, Acosta N, Rodriguez E, Fournet A 1995. Mutagenicity, insecticidal and trypanocidal activity of some Paraguayan Asteraceae. $J$ Ethnopharmacol 45: 35-41.

Baratto L, Lang KL, Vanz DC, Reginatto FH, Oliveira JB, Falkenberg M 2008. Investigação das atividades alelopática e antimicrobiana de Mikania laevigata (Asteraceae) obtida de cultivos hidropônico e tradicional. Rev Bras Farmacogn 18: 577-582.

Barbosa-Filho JM, Vasconcelos THC, Alencar AA, Batista LM, Oliveira RAG, Guedes DN, Falcão HS, Moura MD, Diniz FFM, Modesto-Filho J 2005. Plants and their active constituents from South, Central, and North America with hypoglycemic activity. Rev Bras Farmacogn 15: 392-413.

Bardon A, Cardona L, Catalan CAN, Pedro JR 1996. 15norGuaianolides and germacranolides from Mikania mendocina. Phytochemistry 41: 845-849.

Barroso GM 1958. Mikaniae do Brasil. Rev Arq J Bot Rio de Janeiro 16: 239-333

Berlyn GP, Miksche JP 1976. Botanical microtechnique and cytochemistry. Ames: Iowa State University, p. 121, 276.

Biavatti MW, Koerich CA, Henck CH, Zucatelli E, Martineli FH, Bresolin TB, Leite SN 2004. Coumarin content and physicochemical profile of Mikania laevigata extracts. $Z$ Naturforsch C 59: 197-200.

Bighetti AE, Antonio MA, Kohn LK, Rehder VL, Foglio MA, Possenti A, Vilela L, Carvalho JE 2005. Antiulcerogenic activity of a crude hydroalcoholic extract and coumarin isolated from Mikania laevigata Schultz Bip. Phytomedicine 12: 72-77.

Bishayee A, Chatterjee M 1994. Anticarcinogenic biological response of Mikania cordata: reflections in hepatic biotransformation systems. Cancer Lett 81: 193-200.

Bolina RC, Garcia EF, Duarte MGR 2009. Estudo comparativo da composição química das espécies vegetais Mikania glomerata Sprengel e Mikania laevigata Schultz Bip. ex 
Baker. Rev Bras Farmacogn 19: 294-298.

Botsaris AS 2007. Plants used traditionally to treat malaria in Brazil: the archives of Flora Medicinal. J Ethnobiol Ethnomed 1: 13-18.

Brito CJFA, Alquini Y 1996. A new method for staining botanical material embedded in glycol methacrylate (GMA). Arq Biol Tecnol 39: 949-951.

Corrêa MP 1984. Dicionário das plantas úteis do Brasil e das exóticas cultivadas. Rio de Janeiro, Ministério da Agricultura, IBDF.

Corrêa MFP, Melo GO, Costa SS 2008. Substâncias de origem vegetal potencialmente úteis na terapia da Asma. Rev Bras Farmacogn 18 (Supl.): 785-797.

Cuenca MR, Borkosky S, Catalan CAN, Goedken VL, Draz JG, Herz W 1993. Sesquiterpene lactones of Mikania minima. Phytochemistry 32: 5091-5131.

Do Amaral RR, Arcenio Neto F, Carvalho ES, Teixeira LA, De Araújo GL, Sharapin N, Testa B, Gnerre C, Rocha L 2003. Avaliação da atividade iMAO e anti-bacteriana de extratos de Mikania glomerata Sprengel. Rev Bras Farmacogn 13: 24-27.

Duarte MCT, Figueira GM, Pereira B, Magalhães PM, Delarmelina C 2004. Atividade antimicrobiana de extratos hidroalcólicos de espécies da coleção de plantas medicinais CPQBA/UNICAMP. Rev Bras Farmacogn 14: 6-8.

Fabbri H, Oliveira DCR, Vichnewski W 1997. Diterpenes of Mikania Lindbergii Baker. Biochem Syst Ecol 25: 563564.

Falcão HS, Lima IO, Santos VL, Dantas, HF, Diniz MFFM, Barbosa-Filho, JM, Batista LM 2005. Review of the plants with anti-inflammatory activity studied in Brazil. Rev Bras Farmacogn 15: 381-391.

Feder N, O’Brien TP 1968. Plant microthecnique: some principles and new methods. Amer J Bot 55: 123-142.

Fernandes JB, Vargas VM 2003. Mutagenic and antimutagenic potential of the medicinal plants $M$. laevigata and C. xanthocarpa. Phytother Res 17: 269-273.

Fierro IM, Silva ACB, Lopes CS, Moura RS, Barja-Fidalgo C 1999. Studies on the anti-allergic activity of Mikania glomerata. J Ethnopharmacol 66: 19-24.

Foster AS 1949. Practical plant anatomy. 2. ed. Princeton: D. Van Nostrand.

Francheschi VR, Horner Júnior HT 1980. Calcium oxalate crystals in plants. Bot Rev 46: 361-427.

Holetz FB, Pessini GL, Sanches NR, Cortez DA, Nakamura CV, Filho BP 2002. Screening of some plants used in the Brazilian folk medicine for the treatment of infectious diseases. Mem Inst Oswaldo Cruz 97: 1027-1031.

Johansen DA 1940. Plant microtechnique. New York: McGraw Hill Book, p. 41, 193.

Lentz DL, Clark AM, Hufford CD, Meurer-Grimes B, Passreiter CM, Cordero J, Ibrahimi O, Okunade AL 1998. Antimicrobial properties of Honduran medicinal plants. J Ethnopharmacol 63: 253-263.

Lobitz GO, Tamayo-Castillo G, Merfort I 1997. Diterpenes and sesquiterpenes from Mikania banisteriae. Phytochemistry 46: 161-164

Lobitz GO, Tamayo-Castillo G, Merfort I 1998. Kaurene diterpenes from Mikania vitifolia. Phytochemistry 49: 805-809.

Maiorano VA, Marcussi S, Daher MA, Oliveira CZ, Couto LB, Gomes OA, Franca SC, Soares AM, Pereira PS 2005. Antiophidian properties of the aqueous extract of Mikania glomerata. J Ethnopharmacol 102: 364-370.

Metcalfe CR, Chalk L 1950. Anatomy of dicotyledons: leaves, stem, and woods in relation to taxonomy with notes on economic uses. Oxford: Clarendon Press v. 2.

Milan P, Hayashi AH, Apepezzato-da-glória, B 2006. Comparative leaf morphology and anatomy of three Asteraceae species. Braz Arc Biol Technol 49: 135-144.

Muelas-Serrano S, Nogal JJ, Martinez-Diaz RA, Escario JA, Martinez-Fernandez AR, Gómez-Barrio A 2000. In vitro screening of American plant extracts on Trypanosoma cruzi and Trichomonas vaginalis. J Ethnopharmacol 71: 101-107.

Neves LJ, Sá MFA 1991. Contribuição ao estudo de plantas medicinais Mikania glomerata Spreng. Rev Bras Farm 72: 42-47.

Nunez CV, Amêndola MC, Lago JHG, Roque NF 2004. Diterpene acids from Mikania sp. (Asteraceae). Biochem Syst Ecol 32: 233-237.

O'Brien TP, Feder N, McCully ME 1964. Polychromatic staining of plant cell walls by toluidine blue O. Protoplasma 59: 368-373.

Ohkoshi E, Kamo S, Makino M. Fujimoto Y 2004. ent-Kaurenoic acids from Mikania hirsutissima (Compositae). Phytochemistry 65: 885-890.

Oliveira F 1972. Contribuição para o estudo botânico de Mikania hirsutissima DC. var hirsutissima. II Morfologia externa e anatomia da folha, flor, fruto e semente. Rev Farm Bioquim USP 10: 15-36.

Oliveira F, Akisue G, Akisue MK, Jorge LIF 1986a. Morfodiagnose das folhas e das partes reprodutivas de Mikania laevigata Schultz Bip. ex Baker. Rev Bras Farmacogn 1: 20-34.

Oliveira F, Akisue G, Akisue MK, Jorge LIF 1986b. Morfodiagnose de Mikania laevigata Schultz Bip. Ex Baker - Guaco-do-mato - estudo do axófito. Rev Bras Farmacogn 1: 45-57.

Oliveira F, Akisue G 1991. Fundamentos de farmacobotânica. 2 ed. São Paulo: Atheneu.

Oliveira F, Lúcia M, Garcia LO 1993. Caracterização farmacognóstica da droga e do extrato fluido de mentrasto - Ageratum conyzoides L. Lecta 11: 63-100.

Oliveira F, Rosa FOR, Edna TMK 1999. Estudo farmacognóstico da almécega-da-praia - Mikania conferta Gardn. Lecta 17: 43-68.

Oliveira F, Rodrigues RFO, Bastos DHM, Pereira FH 2000. Caracterização morfohistológica e verificação da atividade microbiológica da espécie vegetal Mikania cordifolia (Lf) Willd. Lecta 18: 33-63. 
Oliveira F, Akisue G, Akisue, MK 2005. Farmacognosia. São Paulo: Atheneu, 2005.

Paul RK, Jabbar A, Rashidi MA 2000. Antiulcer activity of Mikania cordata. Fitoterapia 71: 701-703.

Quintans-Júnior LJ, Almeida JRGS, Lima JT, Nunes XP, Siqueira JS, Oliveira LEG, Almeida RN, Athayde-Filho PF, Barbosa-Filho JM 2008. Plants with anticonvulsant properties - a review. Rev Bras Farmacogn 18 (Supl.): 798-819.

Reis AA, Mendes CC, Ferraz TPL, Roque NF 2003. Terpenes from Mikania hookeriana. Biochem Syst Ecol 31: 10611062.

Reis AA, Ferraz TL, Martins D, Cruz FG, Guedes MLS, Roque NF 2008.Preliminary studies on the volatile constitution of Mikania species. Rev Bras Farmacogn 18 (Supl.): 683-685.

Ritter MR, Miotto STS 2005. Taxonomia de Mikania Willd. (Asteraceae) no Rio Grande do Sul, Brasil. Hoehnea 32: 309-359.

Rodrigues RFIO, Oliveira F, Kato ETM 1996. Morfodiagnose da droga conhecida como cipó-almécega - Mikania malacolepsis Robinson. Rev Farm Bioquim USP 32: $37-$ 44.

Roeser KR 1972. Die Nadel der Schwarzkiefer-Massenprodukt und Kunstwerk der Natur. Mikrokosmos 61: 33-36.

Rüngeler P, Brecht V, Tamayo-Castillo G, Merfort I 2001. Germacranolides from Mikania guaco. Fitoterapia 56: 475-489

Salgado HRN, Roncari AFF, Moreira RRD 2005. Antidiarrhoeal effects of Mikania glomerata Spreng. (Asteraceae) leaf extract in mice. Rev Bras Farmacogn 15: 205-208.

Santos SC, Crueger CL, Steil AA, Kreuger MR, Biavati MW, Wisnievski Junior A 2006. LC characterisation of guaco medicinal extracts, Mikania laevigata and M. glomerata, and their effects on allergic pneumonitis. Planta Med 72: 679-684.

Sass JE 1951. Botanical microtechnique. 2. ed. Ames: Iowa State College, p. 97.

Saúde-Guimarães DA, Faria AR 2007. Substâncias da natureza com atividade anti-Trypanosoma cruzi. Rev Bras Farmacogn 17: 455-465.

Silva RAD 1929. Pharmacopeia dos Estados Unidos do Brasil. São Paulo: Nacional.

Silva RZ, Rios EM, Silva MZ, Leal LF, Yunes RA, Miguel OG, Cechinel Filho V 2002. Investigação fitoquímica e avaliação da atividade antibacteriana da Mikania lanuginosa DC (Asteraceae). Visão Acad 3: 59-64.

Silva CR, Gomes VS, Kulkamp IC, Kanis LA 2008. Método espectroscópico para determinação de cumarina em xarope de Mikania glomerata Sprengel. Rev Bras Farmacogn 18: 594-599.

Soares de Moura R, Costa SS, Jansen JM, Silva CA, Lopes CS, Bernardo-Filho M, Nascimento da Silva V, Criddle DN, Portela BN, Rubenich LM, Araujo RG, Carvalho LC 2002. Bronchodilator activity of Mikania glomerata Sprengel on human bronchi and guinea-pig trachea. $J$
Pharm Pharmacol 54: 249-256.

Souza W 1998. Técnicas básicas de microscopia eletrônica aplicadas às Ciências Biológicas. Rio de Janeiro: Sociedade Brasileira de Microscopia Eletrônica, p. 1-44.

Suyenaga ES, Reche E, Farias FM, Schapoval EE, Chaves CG, Henriques AT 2002. Antiinflammatory investigation of some species of Mikania. Phytother Res 16: 519-523.

Taleb-Contini SH, Santos PA, Veneziani RCS, Pereira MAS, França SC, Lopes NP, DCR Oliveira 2006. Differences in secondary metabolites from leaf extracts of Mikania glomerata Sprengel obtained by micropropagation and cuttings. Rev Bras Farmacogn 16: 596-598.

Veneziani RCS, Camilo D, Oliveira R 1999. Constituents of Mikania glomerata Sprengel. Bichem Syst Ecol 27: 99102.

Zamorano G, Catalan CAN, Díaz JG, Herz W 1994. Germacranolides and sesquiterpeno dilactones from Mikania dusenii. Phytochemistry 37: 187-190. 